\title{
Phase transition dynamics measurements in superheated liquids by monitoring the ejection of nanometer-thick films
}

\author{
Florian Lang ${ }^{\text {a) }}$ and Paul Leiderer ${ }^{\text {b) }}$ \\ University of Konstanz, Universitaetsstr. 10, 78457 Konstanz, Germany \\ Savas Georgiou \\ Institute of Electronic Structure and Laser, Foundation for Research and Technology-Hellas, \\ P.O. Box 1527, GR 71110 Heraklion, Crete, Greece
}

(Received 1 June 2004; accepted 11 August 2004)

\begin{abstract}
The ejection dynamics of nanometer-thick liquid (isopropanol) films from a substrate caused by a phase transition in a superheated layer at the interface are studied. A nanosecond laser pulse is used to heat the substrate and as a result of heat transfer the fluid adjacent to the surface partially reaches a metastable state. Reflectivity and scattered light measurements demonstrate that the liquid is ejected as an intact layer which redeposits on the time scale of a few hundred nanoseconds. Measurements of the ejection dynamics are shown to be a sensitive method to obtain information about the phase transition, such as the relevant time scales and the generated pressure.
\end{abstract}

Laser-induced superheating of liquids in contact with a solid surface and the consecutive phase transition are of interest for a variety of different applications like medical operations $^{1}$ and cleaning of surfaces. ${ }^{2}$ In the past, different experimental studies addressed aspects of the phenomenon such as the threshold fluences for bubble detection in bulk liquid, ${ }^{3}$ the growth velocities of bubbles, ${ }^{4}$ and the generated pressures. ${ }^{5-7}$ A recent overview addressing the topic can be found in Ref. 8.

However, the early stages of the liquid-vapor transition remain unexplored. Investigations of these early stages are essential in order to understand the dynamics of fundamental mechanisms like spinodal decomposition and nucleation phenomena at an interface. Although theoretical models in this regard exist, ${ }^{9-11}$ they are mostly based on questionable thermodynamical equilibrium concepts and have been insufficiently verified by measurements. Direct experimental examinations are challenging because they have to combine a spatial resolution on the nanometer scale with nanosecond or even subnanosecond temporal sensitivity.

As a way to overcome these problems, we examine the lift-off dynamics of nanometer-thick liquid films from a substrate. The ejection dynamics are closely linked to the initial stages of the phase transition providing direct information about the nature of the process. A schematic layout of the setup is shown in Fig. 1.

Thin liquid films are condensed onto the substrate by passing a flow of heated air, saturated with isopropanol vapor. A detailed description of the evaporation and delivery system can be found in Ref. 12. The liquid layer thickness during the condensation is monitored by reflectometry on the millisecond-time scale. Commercially available silicon wafers are employed as substrates due to their high-quality surface flatness.

The phase transition is induced by a $\mathrm{KrF}$ laser pulse $(\lambda=248 \mathrm{~nm}$, full width at half maximum $=29 \mathrm{~ns})$. The laser pulse is absorbed by the silicon and the liquid on

\footnotetext{
${ }^{a)}$ Electronic mail: florian.lang@uni-konstanz.de

${ }^{\text {b) }}$ http://www.uni-konstanz.de/FuF/Physik/Leiderer/index.html
}

top partially reaches a superheated state due to heat conduction across the interface. The subsequent ejection of the liquid from the surface is monitored via temporally resolved reflected and scattered light probe. The reflectometer has a spatial resolution of $2 \mathrm{~nm}$ in the direction perpendicular to the substrate and a temporal sensitivity of $1 \mathrm{~ns}$. Scattered light is measured collaterally by using a telescope and a photomultiplier with a rise time of $1 \mathrm{~ns}$.

Figure 2(a) shows typical signals recorded during one of the experiments. The reflectivity features a steep increase followed by oscillations around the value corresponding to a bare silicon wafer. In view of previous laser flash photography studies, ${ }^{13}$ these oscillations are attributed to the interference effects between an intact liquid layer that is ejected and the substrate surface. However subsequently the pattern smoothens out most likely due to emerging roughnesses at the film surface. Within about $200 \mathrm{~ns}$ the reflectivity signal drops back close to the initial level. By performing double pulse experiments, we could show that the reflectivity signal undergoes a similar sequence as in Fig. 2(a) again when a second laser pulse illuminates the surface shortly after the decrease. Therefore the signal drop is due to redeposition of the liquid layer on the wafer. Hydrodynamic fluid flow from the sides into the area of ejection can be safely excluded due to the velocities significantly above the speed of sound in the liquid that would be required. In the scattered signal a first peak is observed when the pulse irradiates the surface due to scattering from bubbles and also partially to

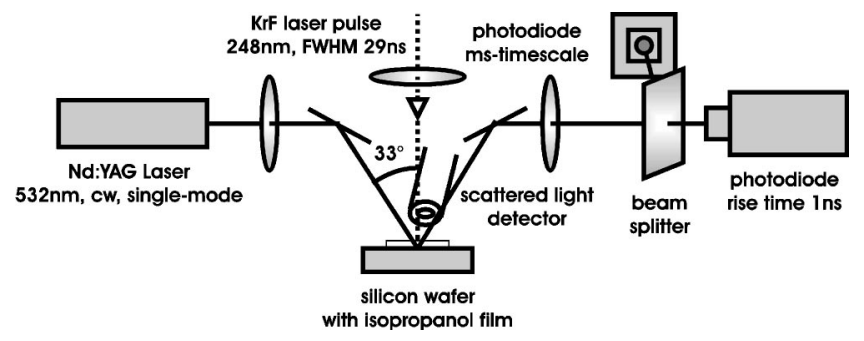

FIG. 1. Schematic drawing of the optical setup. 

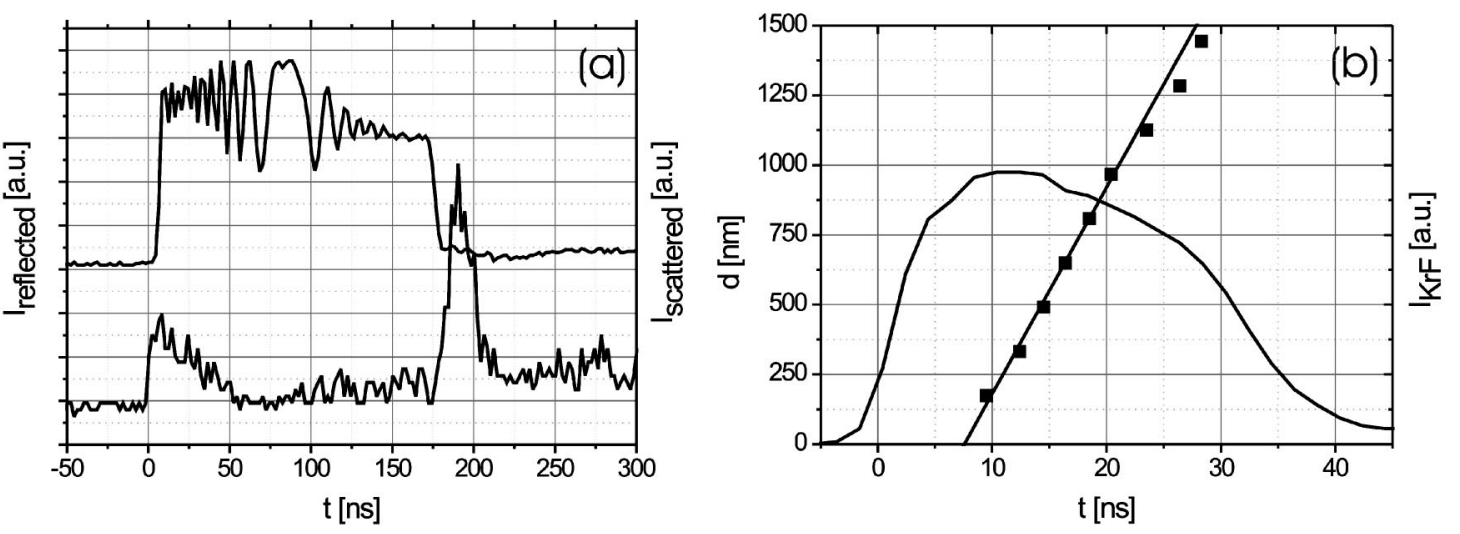

FIG. 2. (a) Reflected (upper curve) and scattered (lower curve) light signal for 90 -nm-thick film (incident fluence $F=150 \mathrm{~mJ} / \mathrm{cm}^{2}$ ). (b) Distance vs time plot of the liquid layer ejection (squares) and temporal profile of the $\mathrm{KrF}$ laser pulse (full line).

imperfect shielding of the detector against UV radiation. Afterwards, a low scattered light level is detected, which supports the picture of an intact layer, which is homogeneous on the probe laser wavelength scale. The second peak occurs at the same time as the drop of the reflectivity to the initial level. Thus, the film disintegrates upon redeposition on the substrate.

The dynamics of the liquid layer ejection are determined from the periodicity of the reflectivity oscillations. For the interspace a refractive index of $n=1$ is assumed. The assumption is reasonable considering that the thickness of the superheated liquid layer is small compared to the lift off distance between the layer and the substrate, which is verified by the simulations described in the following. Figure 2(b) depicts the distance versus time plot of the lift-off process. Using a linear fit of the first data points the ejection time from the surface is found to be $8 \pm 1 \mathrm{~ns}$ after the beginning of the pulse (defined by a trigger level at $25 \%$ of the maximum pulse intensity) and the lift-off velocity is $74 \pm 1 \mathrm{~m} / \mathrm{s}$.

In order to elaborate on a more complete picture of the processes, a one-dimensional heat conduction model is employed to determine the temporal temperature evolution at different spots of the system (Fig. 3). For the simulations the measured laser fluence is adjusted with a scaling factor so that the experimentally required fluence for surface melting

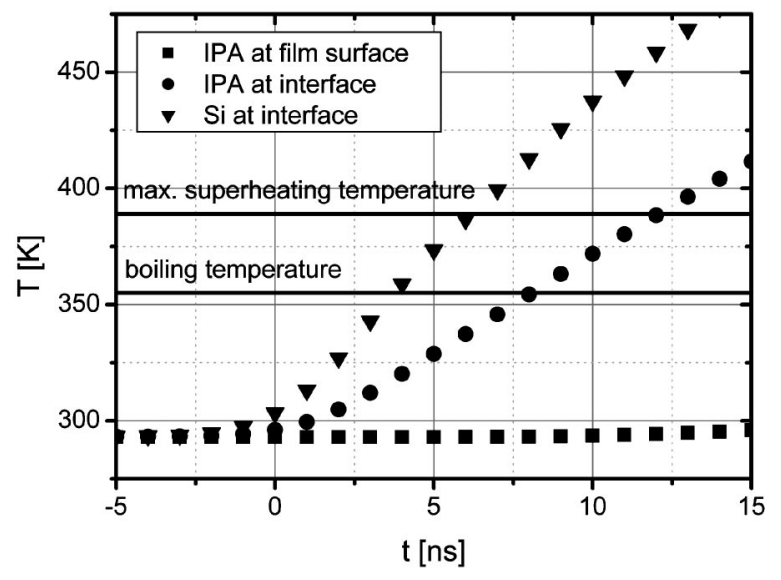

FIG. 3. Calculated temporal temperature evolution at different locations of the system. The simulation was conducted under the conditions of the experiment in Fig. 1. matches the one determined in the calculations. The lower horizontal line in Fig. 3 indicates the boiling temperature of isopropanol $(P=1 \mathrm{~atm})$ and the upper one the highest superheating we experimentally observed so far for isopropanol in contact with a silicon wafer. ${ }^{14}$ The heat transfer coefficient ${ }^{15}$ was set to $10^{7} \mathrm{~W} / \mathrm{m}^{2} \mathrm{~K} .{ }^{14}$ The calculations predict the phase transition to be initiated between 4 and 12 ns after the beginning of the pulse in good agreement with the measured ejection time. In contrast to previous studies, ${ }^{13}$ our experiments prove for the first time that the liquid-vapor phase change responsible for the ejection takes place within at most a few nanoseconds.

Figure 4 displays the complete distance versus time curve for the 90-nm-thick film, including the deceleration and redeposition on the substrate. The data points from the reflectivity oscillation are fitted to a quadratic polynomial. Despite the apparent simplicity of this approach, which assumes a constant acceleration back to the surface, the estimated redeposition time is in excellent agreement with the occurrence of the second peak in the scattered light and the reflectivity drop. Furthermore, the peak of the parabola in Fig. 4 agrees reasonably with the dip in the reflectivity curve, which corresponds to the return point of the film. The force impelling the redeposition is presumably caused by a pressure difference between the areas above and below the liquid layer, which is generated during the rapid ejection. Based on the fitted curve we find a backward acceleration of

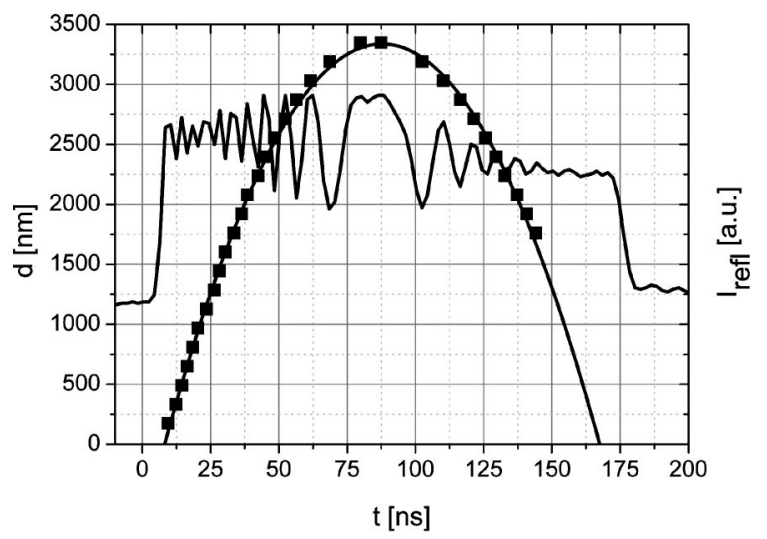

FIG. 4. Measured signals and film ejection dynamics for a $90 \mathrm{~nm}$ liquid laver. 
$(10.5 \pm 0.1) \times 10^{8} \mathrm{~m} / \mathrm{s}^{2}$ corresponding to a pressure difference of $\Delta P=740 \pm 10 \mathrm{hPa}$.

Further information derives from the dependence of the ejection dynamics on the deposited film thickness and the effective fluence of the $\mathrm{KrF}$ laser $F_{\text {eff }}=(1-R(d)) F$, with $R$, $F$, and $d$ denoting reflectivity, incident fluence, and initial film thickness. The ejection time is found to be independent of the film thickness and to decrease linearly as a function of the effective fluence from a value of $8 \mathrm{~ns}$ at $F_{\text {eff }}$ $=50 \mathrm{~mJ} / \mathrm{cm}^{2}$ to $3 \mathrm{~ns}$ at $F_{\text {eff }}=80 \mathrm{~mJ} / \mathrm{cm}^{2}$. Studies on the amount of kinetic energy $E / A=\rho d v^{2} / 2$ and momentum $p / A=\rho d v$ transferred to the liquid per unit area, with $\rho$ denoting the density of isopropanol, show both values to drop with increasing $F_{\text {eff }}$. We attribute this effect to a smaller thickness of the superheated region, if the liquid is heated more rapidly.

Monitoring the ejection dynamics of nanometer-thick films clearly offers a sensitive approach to determine the features of the early phase transition stages in a superheated liquid at an interface. Since the lift-off occurs within a few nanoseconds after the boiling temperature is reached and the typical ejection velocities are between 50 and $100 \mathrm{~m} / \mathrm{s}$, the average initial accelerations are at least on the order of $10^{10} \mathrm{~m} / \mathrm{s}^{2}$. This can be associated to an average pressure difference of about $10^{6} \mathrm{~Pa}$ created due to the phase change. The described measurements, possibly complemented by theoretical considerations such as molecular dynamics simulations, ${ }^{16}$ thus can provide important information for a more detailed understanding of the phase transition in superheated liquids at interfaces.

The authors thank Johannes Graf and Mario Mosbacher for valuable discussions and the staff of IESL-FORTH for the outstanding assistance. Financial support by the Optik
Zentrum Konstanz and the Deutsche Forschungsgemeinschaft (SFB 513). F.L. gratefully acknowledges a fellowship (HPMT-GH-00-00177-13) from the Marie Curie Training Site operating at IESL-FORTH supported by the Human Potential Programme of the European Comission.

${ }^{1}$ A. Vogel and V. Venugopalan, Chem. Rev. (Washington, D.C.) 103, 577 (2003).

${ }^{2}$ W. Zapka, W. Ziemlich, and A. C. Tam, Appl. Phys. Lett. 58, 2217 (1991).

${ }^{3}$ O. Yavas, P. Leiderer, H. K. Park, C. P. Grigoropoulos, C. C. Poon, W. P. Leung, N. Do, and A. C. Tam, Phys. Rev. Lett. 70, 1830 (1993).

${ }^{4}$ O. Yavas, P. Leiderer, H. K. Park, C P. Grigoropoulos, C. C. Poon, W. P. Leung, N. Do, and A. C. Tam, Appl. Phys. A: Solids Surf. 58, 407 (1994).

${ }^{5}$ H. K. Park, D. Kim, C. P. Grigoropolous, and A. C. Tam, J. Appl. Phys. 80, 4072 (1996).

${ }^{6}$ A. Schilling, O. Yavas, J. Bischof, J. Boneberg, and P. Leiderer, Appl. Phys. Lett. 69, 4159 (1996).

${ }^{7}$ O. Yavas, A. Schilling, J. Bischof, J. Boneberg, and P. Leiderer, Appl. Phys. A: Mater. Sci. Process. 64, 331 (1997).

${ }^{8}$ S. Georgiou and A. Koubenakis, Chem. Rev. (Washington, D.C.) 103, 349 (2003).

${ }^{9}$ V. P. Skripov, E. N. Sinitsyn, P. A. Pavlov, G. V. Ermakov, G. N. Muratov, N. V. Bulanov, and V. G. Baidakov, Thermophysical Properties of Liquids in the Metastable (Superheated) State (Gordon and Breach, New York, 1988).

${ }^{10}$ V. P. Carey, Liquid-Vapor Phase-Change Phenomena (Hemisphere, New York, 1992)

${ }^{11}$ P. G. Debenedetti, Metastable Liquids-Concepts and Principles (Princeton University Press, New York, 1996).

${ }^{12}$ F. Lang, M. Mosbacher, and P. Leiderer, Appl. Phys. A: Mater. Sci. Process. 77, 117 (2003).

${ }^{13}$ W. Zapka, Laser Cleaning, edited by B. Luk'yanchuk (World Scientific, Singapore, 2002), Chap. 1.

${ }^{14}$ P. Leiderer, M. Mosbacher, V. Dobler, A. Schilling, O. Yavas, B. Luk'yanchuk, and J. Boneberg, in Ref. 13, Chap. 6.

${ }^{15}$ E. T. Swartz and R. O. Pohl, Rev. Mod. Phys. 61, 605 (1989).

${ }^{16}$ Y. Dou, L. V. Zhigilei, N. Winograd, and B. J. Garrison, J. Phys. Chem. 105, 2748 (2001). 